\title{
Optical spectroscopy of the M 15 intermediate velocity cloud ${ }^{\star}$
}

\author{
J. V. Smoker ${ }^{1}$, F. P. Keenan ${ }^{1}$, N. Lehner ${ }^{2}$, and C. Trundle ${ }^{1,3}$ \\ 1 Astrophysics and Planetary Science Division, Department of Pure and Applied Physics, The Queen's University \\ of Belfast, University Road, Belfast, BT7 1NN, UK \\ 2 Department of Physics and Astronomy, Johns Hopkins University, 3400 North Charles Street, Baltimore, \\ MD 21218, USA \\ 3 Isaac Newton Group, Apartado de Correos 368, Santa Cruz de La Palma, Tenerife 38780, Canary Islands, Spain
}

\begin{abstract}
Received 29 January 2002 / Accepted 14 March 2002
Abstract. We present echelle spectrograph observations in the NaD lines, at resolutions of $6.2-8.5 \mathrm{~km} \mathrm{~s}^{-1}$, for 11 stars located in the line-of-sight to the M 15 intermediate velocity cloud (IVC), which has a radial velocity of $\sim+70 \mathrm{~km} \mathrm{~s}^{-1}$ in the Local Standard of Rest. This cloud is a part of IVC Complex gp. The targets range in magnitude from $m_{V}=13.3-14.8$. Seven of the observed stars are in the M 15 globular cluster, the remaining four being field stars. Three of the observed cluster stars are located near a peak in intensity of the IVC H I column density as observed at a resolution of $\sim 1$ arcmin. Intermediate velocity gas is detected in absorption towards 7 stars, with equivalent widths in $\mathrm{NaD}_{2}$ ranging from $\sim 0.09-0.20 \AA$, corresponding to $\log _{10}\left(N_{\mathrm{Na}} \mathrm{cm}^{-2}\right)$ $\sim 11.8-12.5$, and $\mathrm{NaI} / \mathrm{H}$ I column density ratios (neglecting the $\mathrm{H}$ II component) ranging from $\sim(1-3) \times 10^{-8}$. Over scales ranging from $30 \operatorname{arcsec}$ to 1 arcmin, the Na I column density and the NaI/H I ratio varies by upto 70 per cent and a factor of $\sim 2$, respectively. Combining the current sightlines with previously obtained Na I data from Kennedy et al. (1998b), the Na I/H I column density ratio over cluster sightlines varies by upto a factor of $\sim 25$, when using $\mathrm{H}_{\mathrm{I}}$ data of resolution $\sim 2 \times 1$ arcmin. One cluster star, M $15 \mathrm{ZNG}-1$, was also observed in the Ca I $\left(\lambda_{\text {air }}=4226.728 \AA\right)$ and Ca II $\left(\lambda_{\text {air }}=3933.663 \AA\right)$ lines. A column density ratio $N(\mathrm{Ca}$ I $) / N(\mathrm{Ca}$ II $)<0.03$ was found, typical of values seen in the warm ionised interstellar medium. Towards this sightline, the IVC has a Na I/Ca II column density ratio of $\sim 0.25$, similar to that observed in the local interstellar medium. Finally, we detect tentative evidence for IV absorption in K I $\left(\lambda_{\text {air }}=7698.974 \AA\right)$ towards 3 cluster stars, which have $N(\mathrm{~K} \mathrm{I}) / N(\mathrm{H} \mathrm{I})$ ratios of $\sim 0.5-3 \times 10^{-9}$.
\end{abstract}

Key words. ISM: clouds - ISM: abundances - ISM: individual objects: M15 IVC - ISM: individual objects: complex gp

\section{Introduction}

Fine-scale structure in low velocity interstellar gas, over scales ranging from tens to hundreds of thousands of AU, has been found by many different workers using a variety of techniques, including observations of pulsars, globular clusters and binary stellar systems that act as tracers of the interstellar medium (e.g. Frail et al. 1994; Kennedy et al. 1998a; Andrews et al. 2001). The presence of this structure implies dense clumps of gas in otherwise diffuse sightlines, and large overpressures with respect to the interstellar thermal pressure of $\sim 3000 \mathrm{~cm}^{-3} \mathrm{~K}$ (Welty \& Fitzpatrick 2001). Regardless of whether such clumps exist or are simply an illusion caused by geometric effects

Send offprint requests to: J. V. Smoker, e-mail: jsmoker@eso.org

* Based on observations made with the Apache Point Observatory $3.5-\mathrm{m}$, USA, Very Large Telescope, Chile, and William Herschel Telescope, La Palma, Spain.
(Heiles 1997), the small-scale structures must be explained by any model of the interstellar medium.

For gas at intermediate and high velocities, fewer studies of small-scale structure exist, due to the difficulty in finding relatively bright, closely-spaced probe objects at distances greater than the absorbing material. One obvious location for probe stars is globular clusters. Hence Kerr \& Knapp (1972) performed an absorption-line survey towards many such objects, in particular finding an intermediate-velocity cloud towards M 15, a globular cluster that lies at a distance of $\sim 10 \mathrm{kpc}$ (Carretta et al. 2000). This cloud is a part of the IVC Complex gp (Wakker 2001), a cloud complex located in the line-of-sight towards the HVC Complex GP. Parts of Complex gp are likely to lie at a distance exceeding $0.8 \mathrm{kpc}$ (Little et al. 1994). The distance towards the M 15 IVC is uncertain, although is likely to be less than $3 \mathrm{kpc}$ (Smoker et al. 2001). The study of parts of this cloud is the subject of the current paper.

Previous optical observations of the M 15 IVC include low-resolution Ca II K $\left(\lambda_{\text {air }}=3933.663 \AA\right)$ and Na I D 
$\left(\lambda_{\text {air }}^{\mathrm{D} 2}=5889.950 \AA\right.$ and $\left.\lambda_{\text {air }}^{\mathrm{D} 1}=5895.924 \AA\right)$ absorptionline spectroscopy (Lehner et al. 1999) towards 12 cluster stars, which found that the equivalent widths of the IV component in Ca II K range from 0.05 to $0.20 \AA$, although any variation from star-to-star appeared random and is close to the error in the measurement. Langer et al. (1990) also found variations in IVC Na I D equivalent widths of a factor $\sim 3$ over the cluster face for a handful of stars. Fibre-optic array mapping in the Na I D absorption lines towards the cluster centre (Meyer \& Lauroesch 1999) also found structure visible on scales of $\sim 4 \operatorname{arcsec}$ at a velocity resolution of $\sim 16 \mathrm{~km} \mathrm{~s}^{-1}$. Using empirical relationships between the Na and $\mathrm{H}$ i column densities, Meyer \& Lauroesch (1999) derived values of the H I column density towards the cluster centre of $\approx 5 \times 10^{20} \mathrm{~cm}^{-2}$, which imply a volume density of $\sim 1000 \mathrm{~cm}^{-3}$. This compares with the IVC HI column density towards this point, at a resolution of $\sim 2 \times 1$ arcmin, of $\sim 4 \times 10^{19} \mathrm{~cm}^{-2}$, measured by Smoker et al. (2002) using a combined AreciboWesterbork Synthesis Radio Telescope map. This H I map, shown in Fig. 1, demonstrates that the IV H I column density is actually quite small towards the cluster centre, with a stronger IV column density, still relatively near to the cluster centre, being at $R A \simeq 21^{\mathrm{h}} 29^{\mathrm{m}} 40^{\mathrm{s}}$, Dec $\simeq 12^{\circ} 09^{\prime} 20^{\prime \prime}$. One of the aims of the current study is thus to determine the Na I column density (and variations over small scales), in areas of the IVC where the $\mathrm{H} \mathrm{I}$ is strong. Additionally, the $\mathrm{H}$ I observations show indications of multicomponent structure in velocity, perhaps indicative of cloudlets. Hence it was decided to search for such structure using higher spectral resolution observations than those employed by Meyer \& Lauroesch (1999).

Candidate stars were chosen from the SIMBAD astronomical database where they are all listed as being cluster members. However, a-priori a radial velocity measurement was only available for about half of the stars, and, after analysis of the data, it was found that 4 of the objects were field stars in the line-of-sight towards M 15. The majority of the sample are listed in Battistini et al. (1985), although also included are the single objects KGSY 121 (Kadla et al. 1988), M 15 ZNG-1 (Zinn et al. 1972), and BRO 24 (Brown 1951). The locations of the sample stars are depicted in Fig. 2. Three cluster stars (group "A" in Fig. 2) lie near a peak in the IVC H I column density, with four cluster stars (group "B") at positions where the IVC H I column density is approximately half of this value.

Section 2 describes the observations, Sect. 3 details the data reduction and analysis performed to obtain column densities towards the IVC, Sect. 4 gives the main results, Sect. 5 provides the discussion, and in Sect. 6 the conclusions and suggestions for future work are presented.

\section{Observations}

The observations towards the M 15 IVC were taken during three sessions in June-August 2001, using echelle spectrometers mounted on the Apache Point Observatory

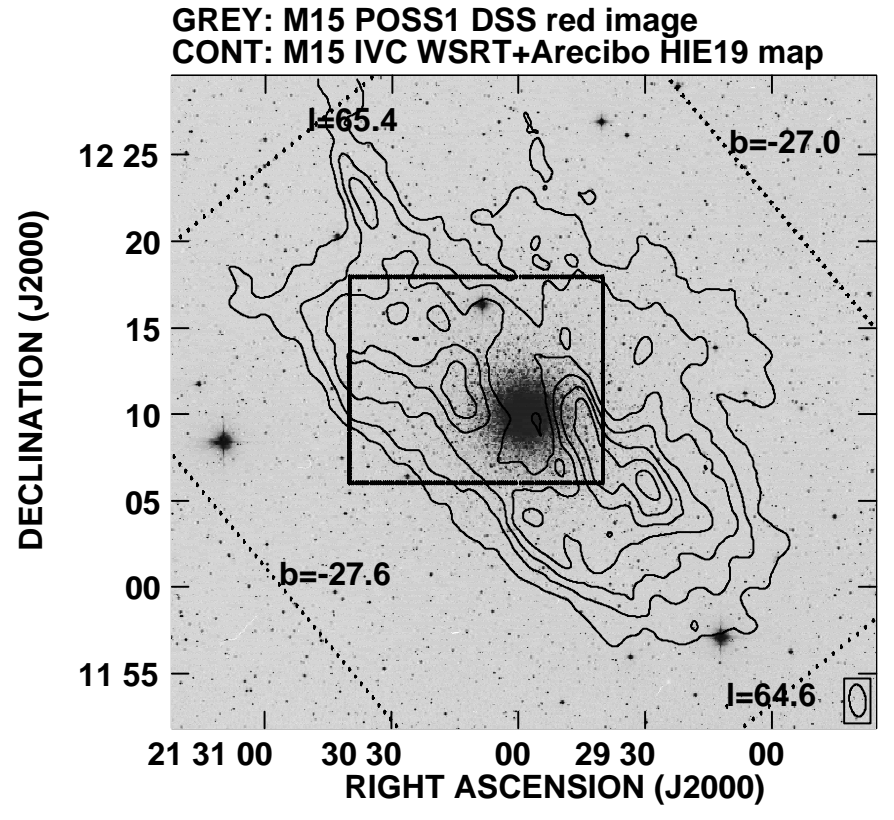

Fig. 1. WSRT plus Arecibo combined H I column density map of the M 15 IVC integrated between +50 and $+90 \mathrm{~km} \mathrm{~s}^{-1}$ in the LSR and at a resolution of $111^{\prime \prime} \times 56^{\prime \prime}$, overlaid on the red POSS-1 digital sky survey image towards M 15 (Smoker et al. 2002). Contour levels are at $N_{\mathrm{HI}}=(2,4,6,8,10,12,14) \times$ $10^{19} \mathrm{~cm}^{-2}$. Lines of constant Galactic longitude and latitude are also plotted on the figure. The rectangle shows the region depicted in Fig. 2.

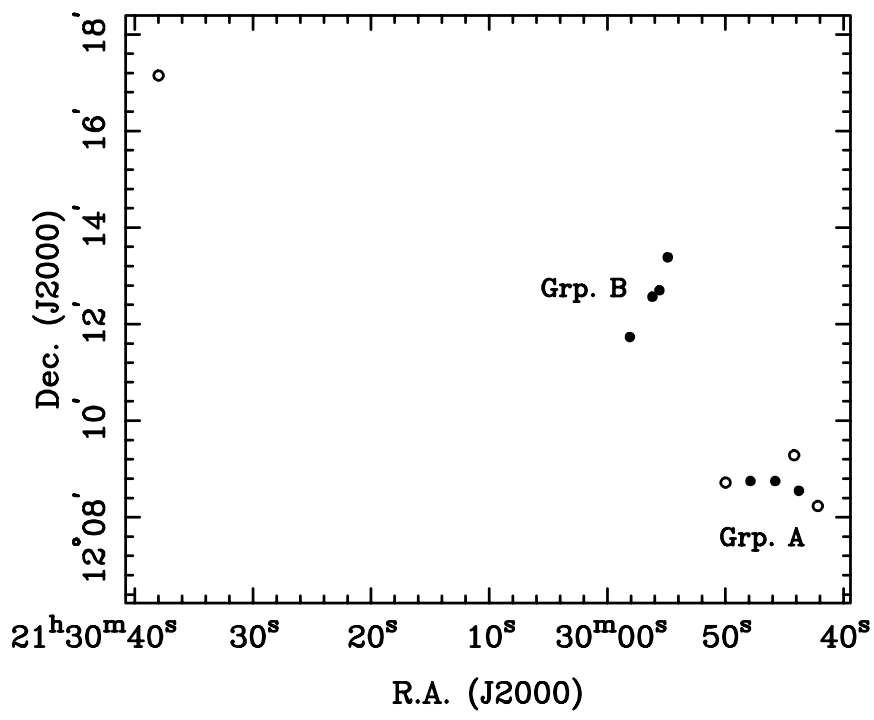

Fig. 2. Spatial distribution of observed sample. Filled circles are cluster stars, open circles are field stars.

3.5-m (APO3.5), 8.2-m Very Large Telescope (UT2; Kueyen), and 4.2-m William Herschel telescope (WHT). The post-AGB star whose spectrum was obtained with the VLT, M 15 ZNG-1, was observed in order to obtain its stellar abundance (Mooney et al. 2002), with the interstellar absorption components described here being detected serendipitously. In order to determine the contribution 
from the night-sky spectrum to the observed data, frequent observations of rapidly-rotating B-type stars were taken which acted as the telluric standards. For the APO3.5 and WHT observations, arc line spectra were taken frequently during the night, and hence the derived velocities are accurate to better than $0.5 \mathrm{~km} \mathrm{~s}^{-1}$, being 3 times the root mean square error on the polynomial fit to the arc-line wavelength calibration. For the VLTobserved star, arcs were only taken at the start and end of each night, thus the wavelength accuracy depends on the stability of the spectrometer. The stellar sample and observing details are listed in Table 1.

\section{Data reduction and analysis}

The APO3.5 and WHT data were reduced using standard methods within IRAF $^{1}$. This included debiasing, wavelength calibration and extraction of the individual orders using DOECSLIT. No flatfielding was performed. For the APO3.5 observations, the limited height of the slit (3.0 arcsec) precluded sky subtraction, and hence we performed a single 750-s exposure on the dark sky to estimate the contribution of the full moon to the night sky brightness. This added between 10-20 per cent to the continuum level for our targets, and was subtracted before the extraction was performed.

For the VLT-observed star, we used data products obtained from the pipeline-calibration which includes wavelength calibration, sky subtraction and flatfielding. The extracted spectra were imported into DIPSO (Howarth et al. 1996), shifted to the Dynamical Local Standard of Rest (LSR) using values obtained from the STARLINK programme RV (Wallace \& Clayton 1996), and normalised by fitting low-order polynomials to the continuum. The radial velocities of the stars were determined by use of the two stellar Na D lines for the cluster objects, and where possible using Ca I $\left(\lambda_{\text {air }}=6717.681 \AA\right)$ for the field stars, with the exception of M 15 ZNG-1 for which we used the Si III features $\left(\lambda_{\text {air }}=4552.622 \AA, 4567.840 \AA\right)$. The broader $\mathrm{H} \delta$ and $\mathrm{H} \beta$ lines were used as a check on the derived velocity. Finally, the normalised spectra were analysed using both the apparent optical depth (AOD) method (Savage \& Sembach 1991) and standard Voigt profile fitting within DIPSO using the ELF and IS programs, for which we used oscillator strengths of $0.6340,0.318,0.6306$ and 0.33919 for the $\mathrm{CaK}, \mathrm{NaD}_{1}, \mathrm{NaD}_{2}$ and K I $\left(\lambda_{\text {air }}=7698.974 \AA\right)$ lines, respectively (Welty et al. 1999). The derived fits were convolved with an instrumental $b$-value of 0.6006 times the FWHM shown in Table 1. The errors on the derived equivalent widths $(E W \mathrm{~s})$ were estimated from a combination of the baseline uncertainty plus the photon noise, and those on the column densities from the $E W$ error, assuming a linear curve of growth.

\footnotetext{
${ }^{1}$ IRAF is distributed by the National Optical Astronomy Observatories, USA.
}

\section{Results}

Figures 3 and 5 show the Na D spectra towards the 11 sample stars. Inspection of the telluric spectra indicate that the only possible contaminating features caused by atmospheric absorption are at $\sim 5896.46 \AA$ and $\sim 5893.83 \AA$, which have equivalent widths of $\sim 5 \mathrm{~m} \AA$, compared to $\sim 90-200 \mathrm{~m} \AA$ for the IV $\mathrm{Na}_{2}$ equivalent widths. Additionally, for both the APO3.5 and WHT spectra, there is some narrow emission present at the wavelengths of the NaD doublet (5889.950 $\AA$ and $5895.924 \AA)$, presumably caused by street lights. This ranged in LSR velocity from $\sim+20$ to $+35 \mathrm{~km} \mathrm{~s}^{-1}$, so was just far enough removed in velocity so as not to contaminate the IV absorption-line at $\sim 65 \mathrm{~km} \mathrm{~s}^{-1}$.

The spectra are composite, and in 7 cases show a stellar component at $\approx-100 \mathrm{kms}^{-1}$, low-velocity (LV) components centred upon $\sim 0 \mathrm{~km} \mathrm{~s}^{-1}$ and intermediate-velocity (IV) components at $\sim+60-70 \mathrm{~km} \mathrm{~s}^{-1}$. Velocities of the M 15 cluster stars range from $\sim-93$ to $-102 \mathrm{~km} \mathrm{~s}^{-1}$, and, at their radial distance from the centre, are each within the range of velocities observed towards M 15 by Drukier et al. (1998): the cluster having a mean LSR velocity of $-97.1 \mathrm{~km} \mathrm{~s}^{-1}$ and velocity dispersion that changes with distance from the centre. For the M 15 cluster stars (excepting M 15 ZNG-1, which is a pAGB), we measure Na I $\mathrm{D}_{1}$ equivalent widths of between 140 and $250 \mathrm{~mA}$, corresponding to A-F spectral types (Gratton \& Sneden 1987; Jaschek \& Jaschek 1987).

Tables 2 and 3 show the results of the profile-fitting to the IV interstellar spectra for the $\mathrm{NaD}$ and $\mathrm{Ca} \mathrm{K}$ lines, respectively. This yields the central velocities, $b$-values and column densities of the IV components. For the three cluster stars in group "A" (near a peak in the IVC H I column density), the derived column densities in the $\mathrm{Na}_{1}$ line are $\sim 0.25$ dex higher than those obtained for the $\mathrm{Na}_{2}$ line, indicating that saturation is occurring in these sightlines, and that the derived column densities are lower limits.

Towards the non-detections, we estimated a $10 \sigma$ limiting equivalent width that would be detected using the current data, $E W_{\text {lim }}$, thus;

$E W_{\text {lim }}=10 \sigma_{\text {cont }} \Delta \lambda_{\text {instr }}$,

where $\sigma_{\text {cont }}$ is $1 /$ (continuum signal-to-noise ratio) and $\Delta \lambda_{\text {instr }}$ is the instrumental full width half maximum $(F W H M)$ in $\AA$. The limiting values of the Na I column densities were estimated by using the $E W_{\text {lim }}$ values estimated above, and $b$-values corresponding to the nearest star for which there were an IV gas detection, assuming that we are on the linear part of the curve of growth. Towards M15 ZNG-1, the above equation was also used to derive upper limits to the column densities of Fe I $\left(\lambda_{\text {air }}=3859.9111 \AA\right), \operatorname{Al}$ I $\left(\lambda_{\text {air }}=3944.0060 \AA\right)$ and KI $\left(\lambda_{\text {air }}=4044.143 \AA\right)$. The $10 \sigma$ upper limits of $\log _{10}\left(N \mathrm{~cm}^{-2}\right)$ of these species of $12.6,11.9$ and 13.1 are high, and are not discussed further in this paper.

Figure 4 shows the KI spectra of the five stars observed with the APO3.5 (the other two observing runs not 
Table 1. Observing parameters and stars observed. Names and magnitudes are from the SIMBAD database and the equinox is J2000. Nomenclature: BBFPnnn: Battistini et al. (1985); BRO 24: Brown (1951); KGSY 121: Kadla et al. (1988); M 15 ZNG-1: Zinn et al. (1972). Res. refers to the spectral resolution of the observations and SNR to the signal-to-noise ratio at a wavelength of $\sim 5900 \AA . N_{\text {IVC }}^{\mathrm{HI}}$ is the H I column density measured at a resolution of $\sim 2 \times 1$ arcmin. Telescope/instrument names; APO/ARC: Apache Point 3.5-m telescope/Chicago Echelle Spectrograph. VLT/UVES: Very Large Telescope (Kueyen)/UV-Visual Echelle Spectrograph. WHT/UES: William Herschel Telescope/Utrecht Echelle Spectrograph.

\begin{tabular}{|c|c|c|c|c|c|c|c|c|c|c|}
\hline Star & $\begin{array}{r}\text { RA } \\
\text { hhmmss.s }\end{array}$ & $\begin{array}{l} \\
\\
\quad, \quad \prime \prime\end{array}$ & $\begin{array}{r}m_{V} \\
\text { (mag.) }\end{array}$ & Tel./Instr. & Obs. Date & $\begin{array}{r}\text { Res. } \\
\left(\mathrm{km} \mathrm{s}^{-1}\right)\end{array}$ & $\begin{array}{r}\text { Time } \\
(\mathrm{s})\end{array}$ & 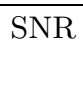 & $\begin{array}{r}N_{\text {IVC }}^{\mathrm{HI}} \\
\left(\times 10^{19} \mathrm{~cm}^{-2}\right)\end{array}$ & $\begin{array}{l}\text { M } 15 \\
\text { star? }\end{array}$ \\
\hline BBFP 493 & 212942.2 & +120814 & 14.27 & $\mathrm{APO} / \mathrm{ARC}$ & 9 Jun. 2001 & 8.5 & 1400 & 30 & 12 & $\overline{\mathrm{No}}$ \\
\hline BBFP 456 & 212943.8 & +120833 & 14.46 & $\mathrm{APO} / \mathrm{ARC}$ & 9 Jun. 2001 & 8.5 & 3000 & 45 & 13 & Yes \\
\hline BBFP 394 & 212944.2 & +120917 & 13.61 & $\mathrm{APO} / \mathrm{ARC}$ & 9 Jun. 2001 & 8.5 & 1400 & 40 & 14 & No \\
\hline BBFP 438 & 212945.8 & +120845 & 13.81 & $\mathrm{APO} / \mathrm{ARC}$ & 8 Jun. 2001 & 8.5 & 4285 & 45 & 12 & Yes \\
\hline BBFP 437 & 212947.9 & +120845 & 13.83 & $\mathrm{APO} / \mathrm{ARC}$ & 9 Jun. 2001 & 8.5 & 1800 & 30 & 11 & Yes \\
\hline BBFP 442 & 212950.0 & +120843 & 12.66 & WHT/UES & 4 Aug. 2001 & 6.8 & 6000 & 40 & 8 & No \\
\hline BBFP 39 & 212954.9 & +121323 & 12.83 & WHT/UES & 2 Aug. 2001 & 6.2 & 2400 & 45 & 6 & Yes \\
\hline BBFP 67 & 212955.6 & +121242 & 13.44 & WHT/UES & 2 Aug. 2001 & 6.2 & 2400 & 30 & 6 & Yes \\
\hline KGSY 121 & 212956.2 & +121234 & 13.02 & WHT/UES & 2 Aug. 2001 & 6.2 & 2400 & 35 & 6 & Yes \\
\hline M 15 ZNG-1 & 212958.1 & +121144 & 14.80 & VLT/UVES & 3 Jul. 2001 & 7.5 & 21600 & 80 & 5 & Yes \\
\hline BRO 24 & 213038.0 & +121709 & 13.3 & WHT/UES & 2 Aug. 2001 & 6.2 & 3600 & 40 & 5 & No \\
\hline
\end{tabular}

Table 2. Results for the IVC NaD absorption-line measurements. Limiting values for the equivalent widths are calculated according to Eq. (1). V corresponds to results obtained using Voigt profile fitting, and D to results obtained using the apparent optical depth method. Upper limits to the column densities are $10 \sigma$ limits calculated according to Eq. (1) assuming a linear curve of growth.

\begin{tabular}{|c|c|c|c|c|c|c|c|}
\hline $\begin{array}{r}\text { Star } \\
\text { Group }\end{array}$ & $\begin{array}{r}v_{\mathrm{LSR}}^{\mathrm{star}} \\
\left(\mathrm{km} \mathrm{s}^{-1}\right)\end{array}$ & $\begin{array}{c}v_{\mathrm{IVC}}^{\mathrm{LSR}}\left(\mathrm{D}_{2}\right) \\
v_{\mathrm{IVC}}^{\mathrm{LSR}}\left(\mathrm{D}_{1}\right) \\
\left(\mathrm{km} \mathrm{s}^{-1}\right) \\
\end{array}$ & $\begin{array}{r}E W_{\text {star }}\left(\mathrm{D}_{2}\right) \\
E W_{\text {star }}\left(\mathrm{D}_{1}\right) \\
(\mathrm{m} \AA)\end{array}$ & $\begin{array}{r}E W_{\text {IVC }}\left(\mathrm{D}_{2}\right) \\
E W_{\text {IVC }}\left(\mathrm{D}_{1}\right) \\
(\mathrm{m} \AA)\end{array}$ & $\begin{array}{l}\log _{10} N\left(\mathrm{D}_{2}\right) \mathrm{V}, \mathrm{D} \\
\log _{10} N\left(\mathrm{D}_{1}\right) \mathrm{V}, \mathrm{D} \\
\left(\log _{10}\left(N \mathrm{~cm}^{-2}\right)\right)\end{array}$ & $\begin{array}{l}b\left(\mathrm{D}_{2}\right) \mathrm{V} \\
b\left(\mathrm{D}_{1}\right) \mathrm{V} \\
\left(\mathrm{km} \mathrm{s}^{-1}\right)\end{array}$ & $\begin{array}{r}N_{\mathrm{NaI}}^{\mathrm{D} 2} / N_{\mathrm{HI}} \\
N_{\mathrm{NaI}}^{\mathrm{D} 1} / N_{\mathrm{HI}} \\
\left(\times 10^{-8}\right) \\
\end{array}$ \\
\hline BBFP 493 & $-24 \pm 3$ & - & - & $<50$ & $<11.48,-$ & - & $<0.3$ \\
\hline$(\mathrm{A})$ & & - & - & - &,-- & - & - \\
\hline BBFP 456 & $-92.6 \pm 0.5$ & $+68.4 \pm 0.3$ & - & $150 \pm 30$ & $>12.13,>12.00$ & $3.5 \pm 0.8$ & 1.0 \\
\hline$(\mathrm{A})$ & & $+68.3 \pm 0.3$ & $136 \pm 15$ & $150 \pm 30$ & $>12.33,>12.26$ & $5.4 \pm 0.6$ & 1.6 \\
\hline BBFP 394 & $-27.5 \pm 1.0$ & - & - & $<40$ & $<11.35,-$ & - & $<0.2$ \\
\hline (A) & & - & - & - &,-- & - & - \\
\hline BBFP 438 & $-99.0 \pm 0.5$ & $+66.2 \pm 0.2$ & - & $190 \pm 30$ & $>12.30,>12.25$ & $5.6 \pm 0.3$ & 1.7 \\
\hline (A) & & $+65.9 \pm 0.1$ & $193 \pm 18$ & $200 \pm 20$ & $>12.55,>12.50$ & $5.5 \pm 0.3$ & 3.0 \\
\hline BBFP 437 & $-102.3 \pm 0.5$ & $+68.5 \pm 0.4$ & - & $200 \pm 20$ & $>12.27,>12.24$ & $5.7 \pm 0.5$ & 1.7 \\
\hline (A) & & $+68.1 \pm 0.4$ & $213 \pm 20$ & $190 \pm 30$ & $>12.56,>12.47$ & $5.3 \pm 0.6$ & 3.3 \\
\hline BBFP 442 & $+19 \pm 5$ & - & - & $<35$ & $<11.33,-$ & - & $<0.3$ \\
\hline (A) & & - & - & - &,-- & - & - \\
\hline BBFP 39 & $-100.0 \pm 0.5$ & $+63.7 \pm 0.4$ & $251 \pm 12$ & $97 \pm 6$ & $12.00 \pm 0.03,12.02 \pm 0.03$ & $4.8 \pm 0.3$ & 1.7 \\
\hline (B) & & $+64.3 \pm 0.3$ & $230 \pm 12$ & $93 \pm 9$ & $12.06 \pm 0.04,12.09 \pm 0.04$ & $5.3 \pm 0.4$ & 1.9 \\
\hline BBFP 67 & $-101.0 \pm 0.5$ & $+63.0 \pm 0.3$ & $226 \pm 14$ & $131 \pm 12$ & $12.02 \pm 0.04,11.93 \pm 0.04$ & $3.6 \pm 0.2$ & 1.7 \\
\hline (B) & & $+63.4 \pm 0.4$ & $222 \pm 14$ & $76 \pm 8$ & $11.98 \pm 0.05,11.94 \pm 0.05$ & $4.0 \pm 0.4$ & 1.6 \\
\hline KGSY 121 & $-96.5 \pm 0.5$ & $+61.0 \pm 0.3$ & $256 \pm 13$ & $129 \pm 10$ & $11.99 \pm 0.03,12.00 \pm 0.03$ & $4.7 \pm 0.4$ & 1.6 \\
\hline (B) & & $+60.9 \pm 0.3$ & $245 \pm 15$ & $79 \pm 6$ & $12.06 \pm 0.03,12.03 \pm 0.03$ & $3.7 \pm 0.4$ & 1.9 \\
\hline M 15 ZNG-1 & $-100.0 \pm 1.0$ & $+64.4 \pm 0.2$ & - & $87 \pm 5$ & $11.80 \pm 0.04,11.79 \pm 0.04$ & $3.8 \pm 0.4$ & 1.3 \\
\hline (B) & & $+63.5 \pm 0.3$ & $21 \pm 4$ & $43 \pm 4$ & $11.79 \pm 0.04,11.79 \pm 0.04$ & $3.7 \pm 0.4$ & 1.2 \\
\hline BRO 24 & $+22 \pm 5$ & - & - & $<35$ & $<11.33,-$ & - & $<0.4$ \\
\hline- & & - & - & - &,-- & - & - \\
\hline
\end{tabular}

covering this wavelength region). In three of the sightlines, there are indications of IV absorption in K I. Although the signal-to-noise of the data are low, we believe that the intermediate-velocity absorption detected towards the cluster stars is likely to be interstellar in nature because; (1) There are no telluric features at the same wavelength as the IV absorption, either in the two field stars or telluric standards; (2) The features are unlikely to be stellar because the centres of two nearby lines (blueshifted by the stellar velocity) of $\mathrm{Nb}$ I $\left(\lambda_{\text {air }}=7703.33 \AA\right)$ and $\mathrm{Cl}$ I $\left(\lambda_{\text {air }}=\right.$ $7702.829 \AA$ ) would be more than $4 \mathrm{~km} \mathrm{~s}^{-1}$ away from the IV absorption peak (at least for the stars BBFP 438 and 


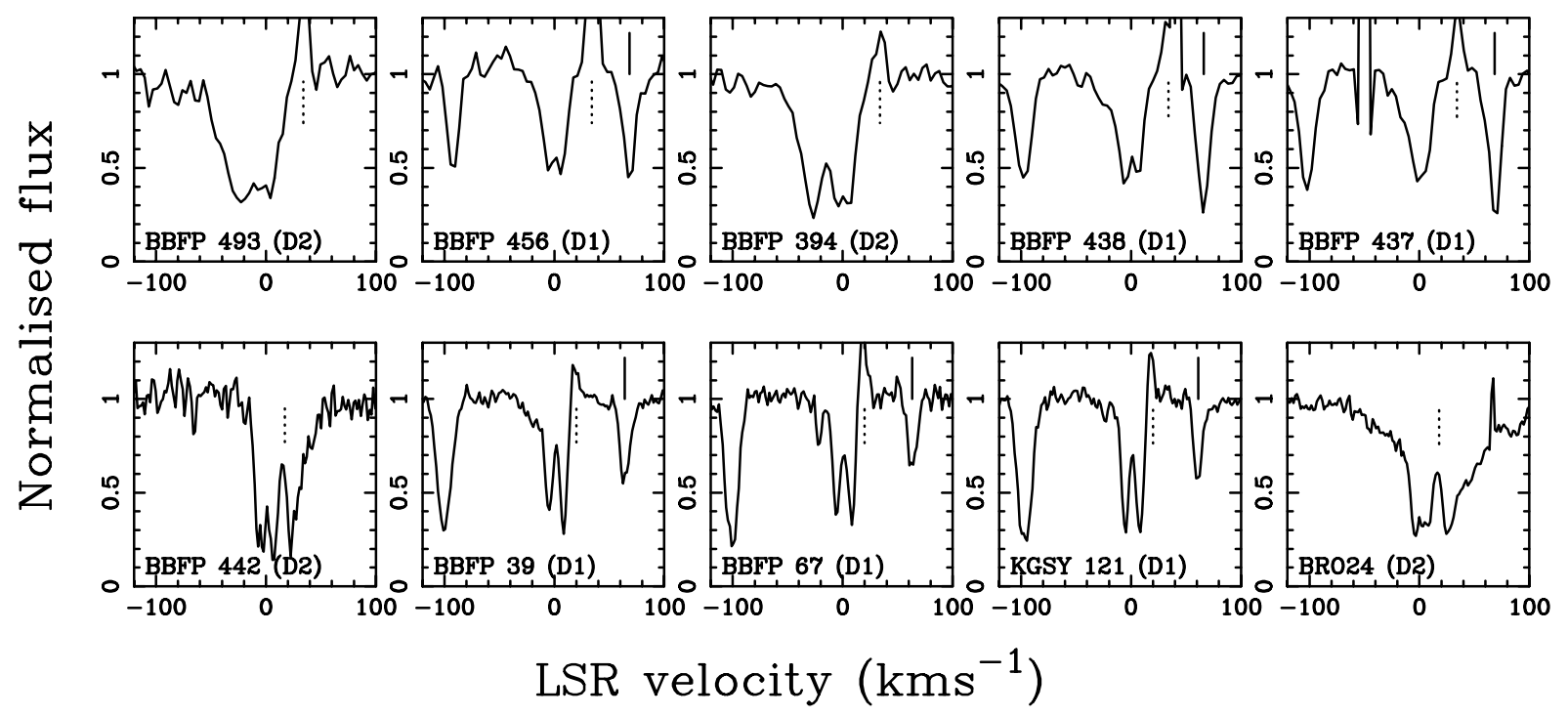

Fig. 3. Na D absorption-line spectra for the whole sample apart from M 15 ZNG-1 (shown in Fig. 5). For IV detections the less-saturated $\mathrm{NaD}_{1}$ line is shown, for non-detections the more sensitive $\mathrm{Na}_{2}$ line is depicted. The abscissa shows the LSR velocity and the ordinate the normalised flux. Solid vertical lines indicate the velocity centroids of the IVC absorption. Dashed vertical lines indicate the presence of emission caused by street lights.

Table 3. Results for the IVC Ca I (4226 $\AA$ ) and Ca II K (3933 $\AA$ ) absorption-line measurements towards M 15 ZNG-1. For the Ca II K-line, a two-component Voigt fit was performed to the data. V corresponds to results obtained using Voigt profile fitting, and $\mathrm{D}$ to results obtained using the apparent optical depth method. Upper limits to the Ca I column densities are $10 \sigma$ limits calculated according to Eq. (1) assuming a linear curve of growth.

\begin{tabular}{rrrrrr}
\hline \hline Transition & $\begin{array}{r}v_{\mathrm{LSR}}^{\text {star }} \\
\left(\mathrm{km} \mathrm{s}^{-1}\right)\end{array}$ & $\begin{array}{r}v_{\mathrm{IVC}}^{\mathrm{LSR}} \\
\left(\mathrm{km} \mathrm{s}^{-1}\right)\end{array}$ & $\begin{array}{r}E W \\
(\mathrm{~mA})\end{array}$ & $\left(\log _{10}\left(N \mathrm{~cm}^{-2}\right)\right) \mathrm{V}, \mathrm{D}$ & $\begin{array}{r}b \mathrm{~V} \\
\left(\mathrm{~km} \mathrm{~s}^{-1}\right)\end{array}$ \\
\hline Ca II K $(3933 \AA)$ & $-100.0 \pm 1.0$ & $52.7 \pm 0.6$ & $6 \pm 1$ & $11.16 \pm 0.07,-$ & $0.6 \pm 4.0$ \\
Ca I $(4226 \AA)$ & $\prime \prime$ & $64.9 \pm 0.1$ & $122 \pm 4$ & $12.40 \pm 0.02,12.36 \pm 0.02$ & $5.3 \pm 0.2$ \\
\hline & $\prime \prime$ & - & $<20$ & $<10.9,-$ & - \\
\hline
\end{tabular}

Table 4. Results for the tentative detection of IV gas in K I $\left(\lambda_{\text {air }}=7698.974 \AA\right)$ towards three M 15 cluster stars. Voigt fitting was used to determine the column densities.

\begin{tabular}{lrrr}
\hline \hline & BBFP 437 & BBFP 438 & BBFP 456 \\
\hline$v_{\mathrm{IVC}}\left(\mathrm{km} \mathrm{s}^{-1}\right)$ & $68.7 \pm 1.0$ & $67.0 \pm 0.5$ & $66.6 \pm 1.0$ \\
$E W_{\mathrm{IVC}}(\mathrm{m} \AA)$ & $28 \pm 8$ & $18 \pm 5$ & $24 \pm 8$ \\
$\log _{10}\left(N_{\mathrm{IVC}}^{\mathrm{KI}} \mathrm{cm}^{-2}\right)$ & $11.20 \pm 0.30$ & $11.10 \pm 0.30$ & $11.15 \pm 0.30$ \\
$N_{\mathrm{KI}} / N_{\mathrm{HI}}\left(\times 10^{-9}\right)$ & 1.4 & 1.0 & 1.1 \\
\hline
\end{tabular}

BBFP 456); (3) The $F W H M$ velocity widths of the IV absorption of $\sim 7-10 \mathrm{kms}^{-1}$ (uncorrected for instrumental broadening: the components are unresolved) are somewhat narrower than the $\mathrm{Na}_{1}$ stellar profiles which have uncorrected $F W H M$ velocity widths from $13-16 \mathrm{~km} \mathrm{~s}^{-1}$. As a species, K I tends to have narrow velocity components, the median width $F W H M$ of 54 Galactic sightlines observed by Welty \& Hobbs (2001) being $\sim 1.2 \mathrm{~km} \mathrm{~s}^{-1}$. Equivalent widths, velocities, and column densities of the IV K I components are shown in Table 4.

\section{Discussion}

\subsection{The distance to the M15 IVC}

We note that in principle the fact that we have obtained non-detections towards 4 field stars in $\mathrm{NaD}$ could enable us to determine a lower-distance limit to this part of the IVC, if the distances to the objects were known. Of the three field stars, two (BBFP 394 and BBFP 493), have $V$ magnitudes in the range delineated by the cluster stars in the current paper (see Buonanno et al. 1983), with BBFP 442 being towards the bright end of the cluster star magnitude range $\left(m_{V}=12.46\right)$. The $(B-V)$ and $(U-B)$ colours of the field stars are $\sim 0.2-0.3 \mathrm{mag}$ and $0.2-0.6 \mathrm{mag}$. bluer, respectively, than the median values for our sample of cluster stars. With the current data, due to the difficulty in ascribing a luminosity class for the field stars, we have not estimated their distances. Additionally, we note that a recent compilation of IVC and HVC Na D column densities by Wakker (2001) has indicated that to be sure of detecting an interstellar absorption in $\mathrm{Na} D$ towards an IVC with $N_{\mathrm{HI}}=10^{19} \mathrm{~cm}^{-2}$, a signal-to-noise ratio (SNR) exceeding $\sim 1000-2000$ is required. Hence, even though the IV H I column densities exceed $10^{20} \mathrm{~cm}^{-2}$ towards three 


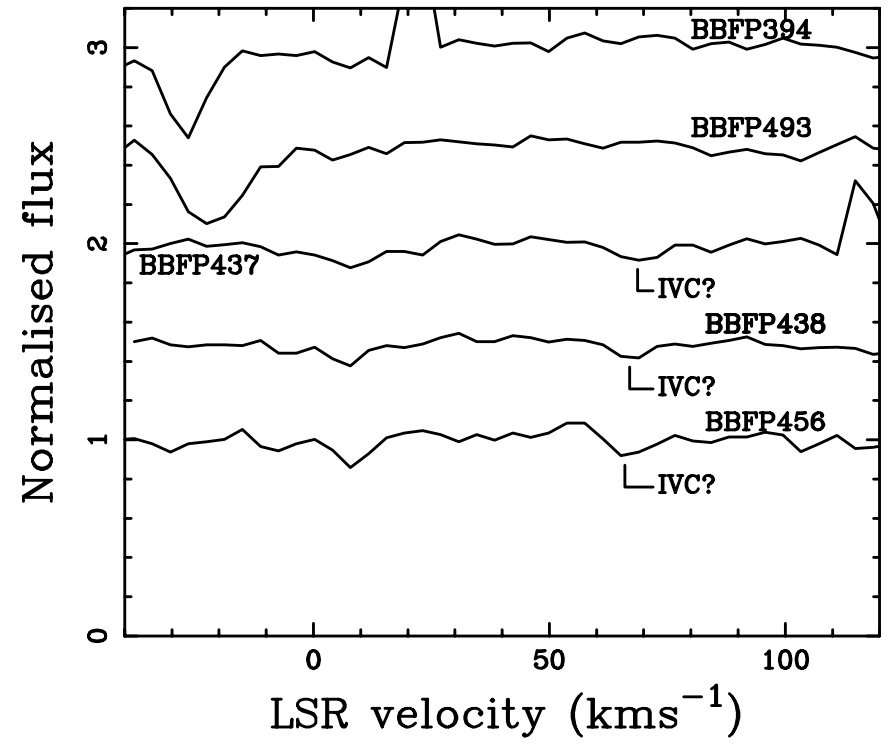

Fig. 4. K I $\left(\lambda_{\text {air }}=7698.974 \AA\right)$ absorption-line spectra for five stars observed with the APO3.5. The top two objects are field stars, with the bottom three being cluster stars. The abscissa shows the LSR velocity and the ordinate the normalised flux, offset in units of 0.5 for clarity.

of our field-star sightlines, the observed SNRs of $\sim 30-40$ in the stars without an IVC detection preclude us from making any statements about the distance to this part of the IVC.

\subsection{Two-component velocity structure}

The presence of two-component velocity structure in IVCs and HVCs indicates the presence of cloudlets, collisions between which have been postulated as a mechanism for star formation in the halo of the Galaxy (Dyson \& Hartquist 1983; although see Christodoulou et al. 1997 and Ramspeck et al. 2001). One of our observed stars, BRO 24, was chosen as a target as it lies near to a position in the IVC H I map where there are indications of twocomponent structure (Smoker et al. 2002). Unfortunately, this star turned out to be a field star. However, towards the object M 15 ZNG-1, we did detect two components (at $\sim+53$ and $+64 \mathrm{~km} \mathrm{~s}^{-1}$ ), in both $\mathrm{CaK}$ and $\mathrm{NaD}$, depicted in Fig. 5, indicating overlapping velocity components in the line-of-sight.

\subsection{Na I and HI column densities and small-scale variations}

Wakker (2001) has summarized the presently-existing absorption-line data for all species reported towards H I clouds, finding that, for LV gas, there is a mean Na I/H I column density ratio of $\sim 5 \times 10^{-9}$, but with a large range exceeding a factor of 10 . Some IVCs (such as the LLIV arch) have similar values to this mean, with others, such as previous measurements towards the M 15 IVC

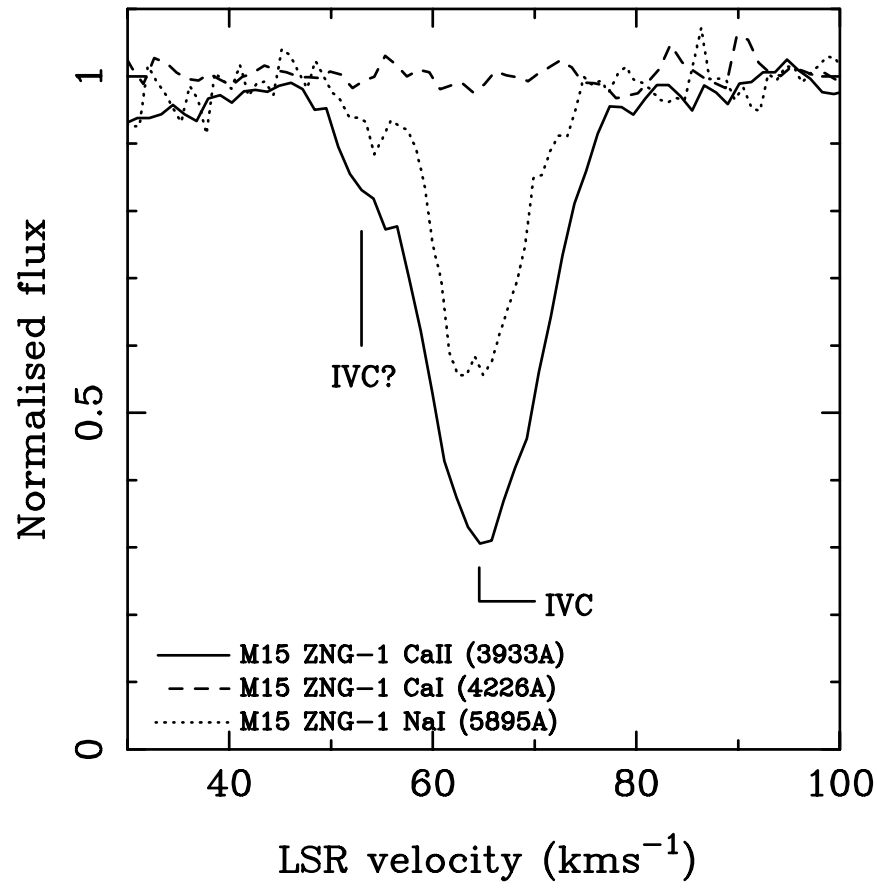

Fig. 5. IVC absorption towards the globular cluster star M 15 ZNG-1, showing possible two-component velocity structure in both $\mathrm{CaK}$ and $\mathrm{NaD}_{1}$ at $\sim+53$ and $+64 \mathrm{~km} \mathrm{~s}^{-1}$. No IVC absorption is evident from the Ca I line at $\lambda_{\text {air }}=4226.728 \AA$. Absorption bluewards of $\sim+40 \mathrm{~km} \mathrm{~s}^{-1}$ originates from the wing of the LV gas.

(Complex gp) showing somewhat higher values, ranging from $(10-160) \times 10^{-9}$ (Langer et al. 1990; Kennedy et al. 1998b; Meyer \& Lauroesch 1999). The Na I/H I column density ratios for the current dataset (based upon $\mathrm{H}$ I data of resolution $\sim 1 \times 2$ arcmin), vary from $\sim(1-3) \times 10^{-8}$, and by as much as a factor of $\sim 2$ in sightlines only separated by 30 arcsec.

Towards the 7 M 15 cluster stars, we derive logarithmic column densities $\left(N\right.$ in $\left.\mathrm{cm}^{-2}\right), \log _{10}(N)$, of between $\sim 11.8-12.5$, the latter value being a lower limit due to saturation. This corresponds to a factor $\sim 6$ variation in column density between parts of group "A" and "B", which compares to a variation in Na I IVC column density of $\sim 16$ over less than an arcminute towards the centre of the cloud (Meyer \& Lauroesch 1999).

For the "A" group of 3 stars, within the errors, two (BBFP 437, 438) have the same value of Na I column density, with BBFP 456 (separated by $\sim 30^{\prime \prime}$ from BBFP 438 ), having a value $\sim 70$ per cent lower than the other two. A rough estimate of the volume density can be made using these data. Assuming that the IVC is at a distance of $1 \mathrm{kpc}$, the separation between BBFP 456 and the other two stars is $\sim 0.15 \mathrm{pc}$ and the approximate transverse cloud size at full width half maximum column density is thus $\sim 0.3$ pc. Following Shaw et al. (1996) and Kennedy et al. (1998b), if this part of the cloud is spherically symmetric, and assuming a $N(\mathrm{NaI}) / N(\mathrm{H} \mathrm{I})$ ratio of $4 \times 10^{-9}$ appropriate to high-latitude clouds (Ferlet et al. 1985), 
the resulting volume density for $\log _{10}\left(N\left(\mathrm{NaI} \mathrm{cm}{ }^{-2}\right)\right)=$ $12.55 \mathrm{is} n_{\mathrm{HI}} \approx 1000 \mathrm{~cm}^{-3}$. Clearly if there is sheet-like structure in the IVC, this volume density is an upper limit, and hence we emphasize that given the difficulty in determining the $N(\mathrm{NaI}) / N(\mathrm{HI})$ ratio and unknown cloud geometry, this implied volume density is very uncertain.

Within the "B" group, three of the stars display similar Na I column density values, apart from M 15 ZNG-1, which is deficient by $\sim 50$ per cent, being separated by $\sim 1$ arcmin from its closest observed companion, KGSY 121. Towards the stars ARP IV-38 and ARP II-75 (Arp 1955), Kennedy et al. (1998b) found $N(\mathrm{Na} \mathrm{I})$ values of $\sim 1.6 \times 10^{12} \mathrm{~cm}^{-2}$ and $2.5 \times 10^{13} \mathrm{~cm}^{-2}$. Using our IVC H I data of resolution $\sim 2 \times 1$ arcmin, these correspond to $N(\mathrm{Na} \mathrm{I}) / N(\mathrm{H} \mathrm{I})$ values of $4 \times 10^{-8}$ and $2.5 \times 10^{-7}$, respectively. The latter value is some $\sim 25$ times greater than observed towards M 15 ZNG-1 and is typical of large variations (often exceeding a factor of 10) that can occur in IVCs/HVCs (Wakker 2001). Possible reasons for this are discussed in Meyer \& Lauroesch (1999).

\subsection{Cloud conditions towards the M 15 ZNG-1 sightline}

Towards the PAGB star M15 ZNG-1, the Hi column density observed at a resolution of $\sim 2 \times 1$ arcmin is $N_{\mathrm{HI}}=5 \times 10^{19} \mathrm{~cm}^{-2}$. Our echelle data of SNR $\sim 80$ cover Ca I $\left(\lambda_{\text {air }}=4226.728 \AA\right)$, Ca II K and the Na I D lines, thus providing some information on the properties of the IV gas by use of these unsaturated features. We determine logarithmic column density values for Ca II, Ca I and $\mathrm{Na}$ I of $\sim 12.4,<10.9$ and 11.8 , respectively. The Ca I/Ca II column density ratio is $<0.03$. We note that this value is consistent with the composite standard model of the photoionised warm interstellar medium of Sembach et al. (2000) (their Table 5), that gives the ionisation fraction $\mathrm{Ca}$ I $/$ Ca II $\sim 0.02$.

Our derived value of $N(\mathrm{Ca}$ II $) / N(\mathrm{HI}) \sim 5 \times 10^{-8}$ for the IVC is within the range found for the high Galactic latitude clouds studied by Albert et al. (1993), namely $(0.3-30) \times 10^{-8} \mathrm{~cm}^{-2}$. However, using the observed $\mathrm{HI}$ column density in combination with the correlation plot of the CaII abundance against Hi column density for IVCs/HVCs from Wakker \& Mathis (2000), a predicted Ca II column density of $\sim 1 \times 10^{-8} \mathrm{~cm}^{-2}$ is obtained. This is $\sim 5$ times smaller than the observed value. Our derived value of $N(\mathrm{Ca}$ II $) / N(\mathrm{HI}) \sim 5 \times 10^{-8}$ implies a depletion of $\sim-1.6$, given a Solar calcium abundance of $2.10 \times 10^{-6}$ and assuming that $\mathrm{Ca}$ II is the dominant ionisation species. However, the model of the photoionised warm interstellar medium of Sembach et al. (2000), predicts that the Ca II/Ca III fraction is $\sim 0.26$. This implies that the Ca III ion is likely to be dominant, particularly as the detection of $\mathrm{H} \alpha$ emission from the cloud (Smoker et al. 2002) indicates that the gas is partially ionised (although perhaps by collisions and not photoionisation; Sembach 1995).
The $N(\mathrm{NaI}) / N(\mathrm{H} \mathrm{I})$ ratio of $\sim 1.3 \times 10^{-8}$ towards this sightline compares with the value derived using the correlation for IVCs/HVCs from Wakker \& Mathis (2000) of $\sim 0.7 \times 10^{-8}$. Combined with the Ca result, this again indicates that this part of Complex gp has high ratios of these elements with respect to $\mathrm{H}$ I when compared with other HVCs and IVCs (cf Wakker 2001). Part of this "overabundance" may be due to the fact that the cloud is partially ionised: Smoker et al. (2002) find that the M 15 IVC has an H $\alpha$ flux exceeding 1 Rayleigh, which, using a reasonable value for the size of the cloud and the calculated emission measure, produces a $\mathrm{H}$ II/H I ratio of $\sim 1-2 \times f^{1 / 2}$, where $f$ is the filling factor of $\mathrm{HII}$ in the IVC.

Towards this sightline, the IVC Na I/Ca II column density ratio is $\sim 0.25 \pm 0.03$. Within the interstellar medium, the range of observed values is from $\sim 0.1-100$; high ratios being found in dense, cold clouds where $\mathrm{Ca}$ is depleted onto dust, and low values occurring in the local warm interstellar medium, where heating of dust grains, for example by shocks, causes grain destruction and a mean $\mathrm{Na}$ I/Ca II ratio of $\sim 0.22$ (Bertin et al. 1993). In the local ISM, some $15-25$ per cent of H I clouds with anomalous velocities exceeding $20 \mathrm{~km} \mathrm{~s}^{-1}$ show such low ratios (see Bertin et al. 1993 and references therein). Grain destruction by shocks is consistent with the observation of strong $\mathrm{H} \alpha$ emission, perhaps caused by collisional ionisation. However, grain destruction does not appear to be total, as Smoker et al. (2002) showed that the $60 \mu$ IRAS map and IVC H I column density map show spatial correspondence, tentatively indicating the presence of some dust in the M 15 IVC. Additionally, large variations in the $\mathrm{Na}$ I/Ca II ratio have been found to occur in components separated by only a few $\mathrm{kms}^{-1}$, and hence Welty et al. (1996) caution against using this ratio as an indicator of either cloud physical conditions or calcium depletions.

\subsection{Tentative KI detections}

There are few observations of K I $\left(\lambda_{\text {air }}=7698.974 \AA\right)$ in IVCs and HVCs (Wakker 2001). Because of its relatively low abundance compared with $\mathrm{Ca}$ or $\mathrm{Na}$, combined with a low ionisation potential, $\mathrm{K} \mathrm{I}$ is normally used to probe relatively dense and cool areas of the interstellar medium. Radio-line $\mathrm{H}$ I observations towards the peaks of the M 15 IVC imply upper limits to the kinetic temperature (in the absence of turbulence and averaged over $\sim 1$ arcmin squared) of less than $\sim 500 \mathrm{~K}$ (Smoker et al. 2002), and H I column densities exceeding $10^{20} \mathrm{~cm}^{-2}$. Hence a priori there was some hope that K I would be detected, given the fact that $\mathrm{Na}$ and $\mathrm{Ca}$ are relatively abundant in the cloud.

The only previously-known detections of K I in IVCs are towards SN 1993J (Vladilo et al. 1994) and towards the M15 IVC by Kennedy et al. (1998b). The latter found $\log _{10}\left(N(\mathrm{KI}) \mathrm{cm}^{-2}\right) \sim 11.4$ for the cluster star M 15 ARP II-75. Using our high-resolution H I map, which has $N_{\text {IVC }}(\mathrm{HI}) \sim 1 \times 10^{20} \mathrm{~cm}^{-2}$, this corresponds to $N(\mathrm{~K} \mathrm{I}) / N(\mathrm{HI}) \sim 2.5 \times 10^{-9}$. Towards another cluster star, 
M 15 ARP II-38, towards which our H I IVC column density map has $N_{\text {IVC }}(\mathrm{HI}) \sim 4 \times 10^{19} \mathrm{~cm}^{-2}$, Kennedy et al. (1998b) obtained an upper limit to $\log _{10}\left(N(\mathrm{~K} \mathrm{I}) \mathrm{cm}^{-2}\right) \sim$ 10.6 , or $N(\mathrm{~K} \mathrm{I}) / N(\mathrm{H} \mathrm{I})<1 \times 10^{-9}$.

These results are similar to our $\mathrm{KI} / \mathrm{HI}$ column density ratios of $\sim 0.5-3 \times 10^{-9}$ (taking into account the large uncertainty in the measurements) for three closelyspaced stars. Based on the uncertainty in our IVC K I detections, we choose not to over-interpret our results. However, we note that both the current data, and that of Kennedy et al. (1998b), display $N(\mathrm{~K} \mathrm{I}) / N(\mathrm{HI})$ values more than 10 times greater than $N(\mathrm{~K} \mathrm{I}) / N\left(\mathrm{H}_{\text {tot }}\right)$ ratios (where $\left.N\left(\mathrm{H}_{\text {tot }}\right)=\mathrm{H} \mathrm{I}+2 \mathrm{H}_{2}\right)$ observed towards Galactic sightlines (Fig. 17 of Welty \& Hobbs 2001). This may be due to a combination of different halo/disc depletions for $\mathrm{K}$, ionisation of the $\mathrm{HI}$ in the IVC (and possible $\mathrm{H}_{2}$ content?), and the fact that our $\mathrm{H}$ I value is derived from data of poorer spatial resolution than the K I data. Finally, we note that the $N(\mathrm{KI}) / N(\mathrm{Na}$ I) values are less than 3 times as large in the IVC as compared with the Galactic results of Welty \& Hobbs (2001), indicating that the high $N(\mathrm{~K} \mathrm{I}) / N(\mathrm{HI})$ value may be caused by one of the factors listed above.

\section{Summary and conclusions}

At a resolution of $\sim 6.2-8.5 \mathrm{~km} \mathrm{~s}^{-1}$, we have observed absorption in $\mathrm{NaI} \mathrm{D}$ at intermediate velocities towards 7 stars within the globular cluster M15. One of these sightlines was also observed in Ca II K, and three in K I. The Na I and Ca II to H I column density ratios lie within the range previously observed in high latitude clouds, although both are in the upper echelons of the observed distribution for IVCs and HVCs, perhaps partly caused by ionisation of $\mathrm{H}$. Variations in the $\mathrm{Na}$ I column density of between 0 to $\sim 70$ per cent towards the IVC have been detected on scales of $\sim 30$ arcsec (or $\sim 0.15$ pc, assuming a cloud distance of $1 \mathrm{kpc}$ ). Over the whole cloud, the $\mathrm{NaI} / \mathrm{H}$ I column density ratio varies by as much as a factor of $\sim 25$.

The Na I/Ca II column density ratio of $\sim 0.25$ is typical of gas in the local warm interstellar medium. This, and the presence of strong $\mathrm{H} \alpha$ emission towards this sightline, raises the possibility that shock ionisation and grain destruction is occurring in the IVC, although the presence of weak IRAS flux implies that grain destruction is not total. Three cluster stars also show tentative detections of KI $(7698.974 \AA)$, with $N(\mathrm{KI}) / N(\mathrm{HI})$ ratios of $\sim 0.5-3 \times 10^{-9}$.

Future observations should concentrate on determining the distance to this part of IVC Complex gp, via spectroscopy of field stars located towards regions of strong IVC H I column density. Towards the Hi peaks, a signal-to-noise exceeding $\sim 20$ in $\mathrm{CaK}$ would be sufficient at a $10 \sigma$ level to interpret a non-detection as being a lower-distance limit, allowing for a factor of 5 variation in the $N(\mathrm{CaII}) / N(\mathrm{HI})$ ratio and with a resolution of $\sim 0.1 \AA$. Finally, UV observations towards the
IVC would much better constrain the gas parameters in the IVC. Although the faintness of the M15 stars precludes this with currently-existing space-based facilities, progress may be possible using the planned Cosmic Origins Spectrograph on the Hubble Space Telescope.

Acknowledgements. Based on observations obtained with the Apache Point Observatory 3.5-meter telescope, which is owned and operated by the Astrophysical Research Consortium, U.S.A., the William Herschel Telescope, operated by the Isaac Newton Group of Telescopes, La Palma, Spain, and at the European Southern Observatory, Cerro Paranal, Chile (programme ID 67.D-0010A). We would like to thank an anonymous referee for useful comments. JVS is grateful to PPARC for financial support and STARLINK for providing computer facilities.

\section{References}

Albert, C. E., Blades, J. C., Morton, D. C., et al. 1993, ApJS, 88,81

Andrews, S. M., Meyer, D. M., \& Lauroesch, J. T. 2001, ApJ, 552,73

Arp, H. C. 1955, AJ, 60, 317

Bertin, P., Lallement, R., Ferlet, R., \& Vidal-Madjar, A. 1993, A\&A, 278, 549

Battistini, P., Bregoli, G., Fusi Pecci, F., Lolli, M., \& Epps Bingham, E. A. 1985, A\&AS, 61, 487

Brown, A. 1951, ApJ, 113, 344

Buonanno, R., Buscema, G., Corsi, C. E., Iannicola, G., \& Fusi-Pecci, F. 1983, A\&AS, 51, 83

Carretta, E., Gratton, R. G., Clementini, G., \& Fusi Pecci, F. 2000, ApJ, 533, 215

Christodoulou, D. M., Tohline, J. E., \& Keenan, F. P. 1997, ApJ, 486, 810

Drukier, G. A., Slavin, S. D., Cohn, H. N., et al. 1998, AJ, 115, 708

Dyson, J. E., \& Hartquist, T. W. 1983, MNRAS, 203, 1233

Ferlet, R., Vidal-Madjar, A., \& Gry, C. 1985, ApJ, 298, 838

Frail, D. A., Weisberg, J. M., Cordes, J. M., \& Mathers, C. 1994, ApJ, 436, 144

Gratton, R. G., \& Sneden, C. 1987, A\&AS, 68, 193

Heiles, C. 1997, ApJ, 481, 193

Howarth, I. D., Murray, J., Mills, D., \& Berry, D. S. 1996, STARLINK, User Note SUN 50, Rutherford Appleton Laboratory/CCLRC

Jaschek, C., \& Jaschek, M. 1987, The classification of stars (Cambridge University Press)

Kadla, Z. I., Gerashchenko, A. N., Strugatskaya, A. A., \& Iablokova, N. V. 1988, Izv. Glav. Astron. Obs., 205, 114

Kennedy, D. C., Bates, B., \& Kemp, S. N. 1998a, A\&A, 336, 315

Kennedy, D. C., Bates, B., Keenan, F. P., et al. 1998b, MNRAS, 297, 849

Kerr, F. J., \& Knapp, G. R. 1972, AJ, 77, 354

Langer, G. E., Prosser, C. F., \& Sneden, C. 1990, AJ, 100, 216

Little, J. E., Dufton, P. L., Keenan, F. P., Conlon, E. S., \& Davies, R. D. 1994, ApJ, 427, 267

Lehner, N., Rolleston, W. R. J., Ryans, R. S. I., et al. 1999, A\&AS, 134, 257

Meyer, D. M., \& Lauroesch, J. T. 1999, ApJ, 520, 103

Mooney, C. J., Rolleston, W. R. J., Keenan F. P., et al. 2002, MNRAS, submitted 
Ramspeck, M., Heber, U., \& Moehler, S. 2001, A\&A, 378, 907 Savage, B. D., \& Sembach, K. R. 1991, ApJ, 379, 245

Sembach, K. R. 1995, ApJ, 445, 314

Sembach, K. R., Howk, J. C., Ryans, R. S. I., \& Keenan F. P. 2000, ApJ, 528, 310

Shaw, C. R., Bates, B., Kemp, S. N., et al. 1996, ApJ, 473, 849

Smoker, J. V., Lehner, N., Keenan, F. P., et al. 2001, MNRAS, 322,13

Smoker, J. V., Haffner, L. M., Keenan, F. P., Davies, R. D., \& Pollacco D. L. 2002, MNRAS, submitted

Vladilo, G., Centurion, M., de Boer, K. S., et al. 1994, A\&A, 291,425
Wakker, B. P., \& Mathis, J. S. 2000, ApJ, 544, 107

Wakker, B. P. 2001, ApJS, 136, 463

Wallace, P., \& Clayton, C. 1996, RV, Starlink, User Note SUN 78, Rutherford Appleton Laboratory/CCLRC

Welty, D. E., Morton, D. C., \& Hobbs, L. M. 1996, ApJS, 106, 533

Welty, D. E., Hobbs, L. M., Lauroesch, J. T., et al. 1999, ApJS, 124,465

Welty, D. E., \& Hobbs, L. M. 2001, ApJS, 133, 345

Welty, D. E., \& Fitzpatrick, E. L. 2001, ApJ, 551, 175

Zinn, R. J., Newell, E. B., \& Gibson J. B. 1972, A\&A, 18, 390 